\title{
Photokeratitis Linked to Metal Halide Bulbs in Two Gymnasiums - Philadelphia, Pennsylvania, 2011 and 2013
}

\author{
Lauren E. Finn, $\mathrm{MPH}^{1}$; Jennifer Gutowski, $\mathrm{MPH}^{1}$; Steve Alles, $\mathrm{MD}^{1}$; Naomi Mirowitz, $\mathrm{MPH}^{1}$; Caroline Johnson, $\mathrm{MD}^{1}$;
} Kevin C. Osterhoudt, $\mathrm{MD}^{2}$; Ami Patel, $\mathrm{PhD}^{1,3}$

In December 2011 and December 2013, the Philadelphia Department of Public Health (PDPH) received separate reports of clusters of photokeratitis linked to gymnasium events. Photokeratitis, a painful eye condition resulting from unprotected exposure to ultraviolet radiation, has previously been linked to metal halide lamps with broken outer envelopes $(1,2)$. To investigate the cause of these clusters and further characterize patients with photokeratitis, PDPH administered questionnaires to potentially exposed persons, established a case definition, and conducted environmental assessments of both gymnasiums. Because event attendee registration information was available, a cohort study was conducted to evaluate the 2011 cluster of 242 persons who met the photokeratitis case definition. A case-series investigation was conducted to evaluate the 2013 cluster of 20 persons who met the photokeratitis case definition for that event. These investigations indicated that Type R metal halide bulbs with broken outer envelopes found in both gymnasiums were the probable cause of the photokeratitis. The Food and Drug Administration has made a number of recommendations regarding the use of metal halide bulbs in facilities where bulbs are at elevated risk for breaking, such as schools and indoor sports facilities (3). Because Type R metal halide lamps do not self-extinguish once the outer envelope is broken, these bulbs should be removed from settings with a high risk for outer envelope rupture, such as gymnasiums, or should be placed within enclosed fixtures. In instances where these bulbs cannot be exchanged for self-extinguishing lamps, Type $\mathrm{R}$ lamps with a broken outer envelope should be replaced immediately to limit exposure to ultraviolet radiation. A broken outer envelope can be detected by the presence of glass on the floor, or visual examination of the bulb when the power is turned off. A broken outer envelope is difficult to detect when the lamp is emitting light.

\section{Investigation of 2011 Outbreak}

During December 4-6, 2011, a total of 127 persons sought care at local emergency departments (EDs) and physicians' offices for eye irritation, including burning, redness, tearing, and foreign-body sensation. Active surveillance by the Poison Control Center (PCC) at The Children's Hospital of Philadelphia alerted PDPH, and it was learned that all patients had attended a 9-hour cheerleading event at a local high school gymnasium on December 4. The ED that treated 12 of the first patients reported that their symptoms were consistent with acute conjunctivitis caused by a chemical irritant. Philadelphia police, fire, and health departments evaluated the gymnasium, including reviewing video footage and testing for hazardous chemicals. The Hazardous Materials Unit of the Philadelphia Fire Department tested for the presence and concentration of toxic industrial chemicals, radioactive materials, volatile organic compounds, and hazardous gases. Wipe testing was performed to ascertain the presence of tear gas residues and quaternary ammonium compounds. All tests for chemical agents were negative; however, a metal halide bulb with a broken outer envelope was found in the gymnasium ceiling. Through video verification, this bulb was determined to have been operational both at the time of environmental assessment and during the cheerleading competition and was identified as the likely source of eye irritation.

On December 7, a web-based survey was conducted to ascertain attendees' exposure and symptom histories. The survey was e-mailed to all cheerleading team coaches, who then distributed the survey link to the parents of each squad member. The survey requested information for all event attendees in each household. A case of photokeratitis was defined as the occurrence of two or more acute eye symptoms in a person who attended the competition.

Surveys were completed by 760 persons, representing approximately $75 \%$ of attendees. Among respondents, 242 $(32 \%)$ met the case definition. Acute eye symptoms reported included burning eyes $(93 \%)$, red eyes $(86 \%)$, tearing eyes (76\%), and foreign body sensation (74\%) (Table 1). The median interval between exposure and symptom onset was 9 hours (range $=0-72$ hours). The median age of symptomatic persons was 29 years (range $=2$ weeks -72 years). Among the 127 persons who sought care, 99 (78\%) went to EDs. Risk for becoming a case was higher among those who sat in the bleachers for $\geq 2$ hours and was lower for persons wearing contact lenses or eyeglasses (Table 2). Affected persons spent more time in the gymnasium (mean $=5$ hours) than nonaffected persons $($ mean $=3$ hours $)(\mathrm{p}<0.01)$. 
TABLE 1. Predominant clinical symptoms reported by photokeratitis patients who attended events in two gymnasiums - Philadelphia, Pennsylvania, 2011 and 2013

\begin{tabular}{|c|c|c|}
\hline & $\begin{array}{c}\text { Cheerleading } \\
\text { competition, } \\
\text { December 4, } 2011 \\
(\mathrm{n}=242)^{*} \\
\end{array}$ & $\begin{array}{c}\text { Recreation center, } \\
\text { December 23-30, } 2013 \\
(\mathrm{n}=20)^{\dagger}\end{array}$ \\
\hline Symptom & No. (\%) & No. (\%) \\
\hline Burning eyes & $225(93)$ & $20(100)$ \\
\hline Red eyes & $207(86)$ & $19(95)$ \\
\hline Tearing eyes & $183(76)$ & $19(95)$ \\
\hline Foreign body sensation & $180(74)$ & $13(65)$ \\
\hline Blurry vision & $123(51)$ & $16(80)$ \\
\hline Eyelid swelling & $102(42)$ & $8(40)$ \\
\hline Skin irritation & $49(20)$ & $4(20)$ \\
\hline
\end{tabular}

* Cohort study.

+ Case series.

\section{Investigation of 2013 Outbreak}

On December 28, 2013, seven persons were evaluated at a local Philadelphia ED for symptoms of photokeratitis, including burning eyes, red eyes, and tearing, following activities at a recreation center gymnasium. PCC reported this cluster of eye irritation to PDPH. During the following week, three additional persons with photokeratitis linked to the same recreation center gymnasium were seen at a local eye hospital. PDPH staff conducted an environmental health assessment at the recreation center. The gymnasium included a basketball court and one set of spectator bleachers, illuminated by 10 industrial ceiling lamps. No evidence of a chemical agent was found. A functioning metal halide lamp with a broken outer envelope was identified in the gymnasium. Informed by the 2011 photokeratitis outbreak, PDPH identified a compromised metal halide lamp as the source of eye irritation in these 10 patients.

A case of photokeratitis was defined as the occurrence of two or more symptoms of acute eye irritation in a person who had engaged in activities at the gymnasium during December 23-30, 2013. As a result of the strong epidemiologic link between the reported cases and this gymnasium, PDPH conducted additional case-finding through a search of ED chief complaint data for the terms "conjunctivitis," "eye injury," "pink eye," "eye emergency," "red eyes," and "burning eyes," and identified 12 additional persons with symptoms suggesting photokeratitis. Further case-finding entailed asking photokeratitis patients to provide names and contact information for other persons present at the recreation center. Eighteen additional exposed persons were identified in this manner for a total of 40 potential patients. Seven persons identified through ED chief complaint data and 12 persons identified through interviews with photokeratitis patients could not be contacted.
TABLE 2. Analysis of two risk factors for photokeratitis among attendees in a gymnasium at a cheerleading competition Philadelphia, Pennsylvania, 2011

\begin{tabular}{|c|c|c|c|c|}
\hline \multirow[b]{2}{*}{ Risk factor } & \multirow{2}{*}{$\begin{array}{c}\begin{array}{c}\text { Cases } \\
(\mathrm{n}=242)\end{array} \\
\text { No. }(\%)\end{array}$} & \multirow{2}{*}{$\begin{array}{c}\begin{array}{c}\text { Noncases } \\
(\mathrm{n}=518)\end{array} \\
\text { No. }(\%)\end{array}$} & \multirow{2}{*}{$\begin{array}{c}\text { Total } \\
(\mathrm{N}=760)\end{array}$} & \multirow{2}{*}{$\begin{array}{l}\text { Relative risk } \\
\quad(95 \% \mathrm{Cl})\end{array}$} \\
\hline & & & & \\
\hline \multicolumn{5}{|c|}{ Time sitting in bleachers } \\
\hline$\geq 2$ hours & $166(69)$ & $267(52)$ & 433 & $1.65(1.31-2.08)$ \\
\hline$<2$ hours & $76(31)$ & $251(48)$ & 327 & Referent \\
\hline \multicolumn{5}{|c|}{ Use of eyeglasses or contact lenses } \\
\hline Yes & $39(16)$ & $132(25)$ & 171 & $0.66(0.49-0.89)$ \\
\hline No & $203(84)$ & $386(75)$ & 589 & Referent \\
\hline
\end{tabular}

Abbreviation: $\mathrm{Cl}=$ confidence interval.

A telephone-based questionnaire to ascertain clinical symptoms and recreation center exposure history was administered to 21 contacted suspected patients. The exposure period among interviewed persons was December 23-30, 2013. A total of 20 patients met the case definition. Eighteen reported playing basketball in the gymnasium, and two were spectators. Eighteen patients were male, and the median age was 26 years (range $=14-57$ years). Seventeen of the 20 patients sought care at EDs. Length of gymnasium exposure ranged from 30 minutes to 4 hours. Predominant acute eye symptoms included burning (100\%), redness (95\%), and tearing (95\%) (Table 1). Four patients also reported peeling or flaking skin. Symptom duration ranged from 3 hours to 9 days. Among 16 patients, symptoms resolved 1-3 days after onset. In 10 of the 17 photokeratitis patients who sought health care, infectious conjunctivitis (five patients), dry eyes (three), or allergic reactions (two) were the initial diagnoses.

\section{Discussion}

Metal halide lamps produce an electric arc that travels through a mixture of mercury and metal halide gases, generating an intense white light. Commonly used for overhead lighting, each lamp has a coated outer glass bulb surrounding the arc tube, which serves to filter out ultraviolet light. Broken metal halide lamps pose a risk for photokeratitis among exposed persons. In the first cluster reported, 242 persons developed photokeratitis following exposure to a single compromised metal halide bulb, and in the second, as few as 30 minutes of exposure to a metal halide bulb with a broken outer envelope resulted in 20 photokeratitis cases.

The link between photokeratitis and metal halide bulbs has been reported previously. In February 2002, the North Carolina Division of Public Health investigated 13 cases of eye and skin burns in a school gymnasium that were presumed to be caused by a broken metal halide lamp (1), and in 


\section{Summary}

What is already known about this topic?

Exposure to broken metal halide bulbs can result in skin and eye irritation, including photokeratitis, particularly in settings such as sports facilities where balls or other objects are routinely thrown.

What is added by this report?

In December 2011 and December 2013, separate clusters of photokeratitis were linked to gymnasium events in Philadelphia where broken metal halide bulbs were in use. Predominant symptoms included burning or red eyes, tearing eyes, foreign body sensation, blurry vision, eyelid swelling, and skin irritation.

What are the implications for public health practice?

Broken metal halide bulbs pose a continuing risk for photokeratitis, particularly in high-risk settings such as gymnasiums. Improved provider education regarding the clinical presentation and exposures associated with photokeratitis might prevent misdiagnosis and promote the expedient identification of future exposures. Facility managers need to be trained to examine bulbs for breaks and replace them immediately upon bulb rupture.

2003, the Tennessee Department of Health identified three separate clusters of photokeratitis and skin burns linked to damaged metal halide bulbs in school and municipal gymnasiums (2). Recommendations from both the Food and Drug Administration and the National Electrical Manufacturers Association cite broken metal halide bulbs as a cause of skin burns and eye irritation, particularly in settings such as sports facilities where balls or other objects are routinely thrown $(3,4)$.

Although the acute symptoms of photokeratitis resolve within a few days, the association with long-term sequelae such as corneal neuropathy is not known. Educational strategies aimed at both facilities management personnel and health care providers are needed. Facilities management personnel should be made aware of the dangers posed by operational metal halide bulbs with broken outer envelopes. Ideally, per Food and Drug Administration recommendations, all nonextinguishing Type $\mathrm{R}$ metal halide bulbs should be replaced with self-extinguishing Type $\mathrm{T}$ bulbs to avoid unintentional exposure to ultraviolet radiation without the protective outer envelope (3). However, Type $T$ bulbs can take as long as 15 minutes to self-extinguish after the outer envelope has been broken. If a lamp of any type breaks, persons should leave the area immediately (5). When replacement with Type $\mathrm{T}$ bulbs is not feasible, a broken bulb must be removed immediately and replaced with an unbroken bulb. Type $\mathrm{R}$ bulbs should not be used in high-risk settings, and should be placed within protective casings to reduce the risk for outer envelope rupture. Facility managers need to be trained to examine bulbs for breaks and replace them immediately upon bulb rupture. A suspected broken bulb should never be examined when the lamp is turned on.

During both of the photokeratitis outbreaks described in this report, patient symptoms were confused with those resulting from a chemical exposure, infectious conjunctivitis, dry eye conditions, or allergic reactions. During the 2013 outbreak, early identification of photokeratitis symptoms could have accelerated identification and removal of the broken metal halide bulb, thus preventing continued exposure of persons using the gymnasium later in the week. In both reported clusters, collaboration between PCC and PDPH facilitated the identification of the cause of eye irritation and an appropriate public health response.

The findings in this report are subject to at least three limitations. First, for both events, clinical symptoms and exposure duration were unverified and dependent on patient recall, which might have resulted in recall or reporting bias. Second, during the 2013 outbreak, lists of gymnasium users and their contact information were not maintained; therefore, it was not possible to identify all of the exposed persons. Finally, because no asymptomatic exposed persons were identified for the 2013 event, measures of risk could not be calculated.

Extensive collaboration between clinicians and interagency partners facilitated rapid identification, reporting, and investigation of these clusters, and permitted determination of the sources of photokeratitis. Improved provider education regarding the clinical presentation and exposures associated with photokeratitis might reduce misdiagnosis and promote the expedient identification of future exposures.

\section{Acknowledgments}

Claire Newbern, PhD, John Faherty, MA, Philadelphia Department of Public Health, Pennsylvania.

\footnotetext{
${ }^{1}$ Division of Disease Control, Philadelphia Department of Public Health, Pennsylvania; ${ }^{2}$ The Poison Control Center at The Children's Hospital of Philadelphia, Pennsylvania; ${ }^{3}$ Division of State and Local Readiness, Office of Public Health Preparedness and Response, CDC.

Corresponding author: Lauren E. Finn, lauren.finn@phila.gov, 215-685-6742.
}

\section{References}

1. Mazzuckelli LF, MacDonald PDM, Langley RL, Howell RJ. Erythema and conjunctivitis: investigation of an outbreak in a school gymnasium caused by unintentional exposure to ultraviolet radiation from metal halide lamps. J Occup Environ Hyg 2007;4:D46-9. http://dx.doi. org/10.1080/15459620701246513

2. Kirschke DL, Jones TF, Smith NM, Schaffner W. Photokeratitis and UV-radiation burns associated with damaged metal halide lamps. Arch Pediatr Adolesc Med 2004;158:372-6. http://dx.doi.org/10.1001/ archpedi.158.4.372 
3. Food and Drug Administration. Ultraviolet radiation burns from high intensity metal halide and mercury vapor lighting remain a public health concern: notice to schools and other indoor, all-purpose facilities where light bulbs are subject to damage. Silver Spring, MD: Food and Drug Administration; 2009. http:/www.fda.gov/Radiation-EmittingProducts/ RadiationSafety/AlertsandNotices/ucm116540.htm
4. National Electrical Manufacturers Association. Recommendations for the care and maintenance of high intensity metal halide and mercury lighting in schools. Rosslyn, VA: National Electrical Manufacturers Association; 2003. https://www.nema.org/Policy/Environmental-Stewardship/Lamps/ Documents/NEMA\%20Recommendations.Jan.\%202003.pdf

5. New Jersey Department of Environmental Protection. Mercury vapor lamps, question and answer. Trenton, NJ: New Jersey Department of Environmental Protection. http://www.nj.gov/dep/rpp/nrs/qamvl.htm 